\title{
Crown closure segmentation on wetland lowland forest using the mean shift algorithm
}

\author{
Beni Iskandar, I Nengah Surati Jaya, Muhammad Buce Saleh \\ Department of Forest Management, Faculty of Forestry and Environment, IPB University, Campus IPB Dramaga, Bogor, \\ Indonesia
}

\begin{abstract}
Article Info
Article history:

Received Oct 8, 2020

Revised Sep 6, 2021

Accepted Sep 16, 2021

Keywords:

Crown closure

Mean-shift

Segmentation

Wetland lowland forest

ABSTRACT

The availability of high and very high-resolution imagery is helpful for forest inventory, particularly to measure the stand variables such as canopy dimensions, canopy density, and crown closure. This paper describes the examination of mean shift (MS) algorithm on wetland lowland forest. The study objective was to find the optimal parameters for crown closure segmentation Pleiades-1B and SPOT-6 imageries. The study shows that the segmentation of crown closure with the red band of Pleiades-1B image would be well segmented by using the parameter combination of (hs: 6, hr: 5, M: 33) having overall accuracy of $88.93 \%$ and Kappa accuracy of $73.76 \%$, while the red, green, blue (RGB) composite of SPOT-6 image, the optimal parameter combination was $(h s: 2, h r: 8, M: 11)$, having overall accuracy of $85.72 \%$ and kappa accuracy of $68.33 \%$. The Pleiades-1B image with a spatial resolution of $(0.5 \mathrm{~m})$ provides better accuracy than SPOT-5 of $(1.5 \mathrm{~m})$ spatial resolution. The differences between single spectral, synthetic, and RGB does not significantly affect the accuracy of segmentation. The study concluded that the segmentation of high and very high-resolution images gives promising results on forest inventory.
\end{abstract}

This is an open access article under the CC BY-SA license.

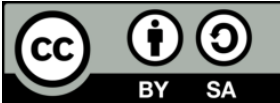

\section{Corresponding Author:}

I Nengah Surati Jaya

Department of Forest Management

Faculty of Forestry and Environment, IPB University

Ulin Road, IPB University, Dramaga, Bogor, Indonesia

Email: ins-jaya@apps.ipb.ac.id

\section{INTRODUCTION}

The extensive and intensive use of satellite remote sensing data in Indonesia to support natural resource management, especially forest monitoring has been started since 1990 using low spatial resolution Landsat imagery $(30-80 \mathrm{~m})$ [1]. Since the advent of high spatial resolution imageries such as IKONOS (1 m panchromatic) image in 1999, followed by QuickBird $(0.6 \mathrm{~m})$ band panchromatic in 2001, OrbView (1 m) band panchromatic in 2011, SPOT-6 $(1.5 \mathrm{~m})$ band panchromatic, and Pleiades-1B $(0.5 \mathrm{~m})$ band panchromatic, there had been a shift in the use of imagery towards operational practical activities such as forest inventory to assess the timber standing stock [1], [2]. In 2016, the Ministry of Environment and Forestry has issued a regulation to formalize the use of high- and very-high resolution imageries for periodic comprehensive forest (PCFI) performed by each concession holder. The image analyses for the PCFI were mostly done by using visual interpretation.

Forest inventory is an activity to collect information about forest conditions [3]. Inventory of wetland lowland forest ecosystems is quite challenging and not efficient, if all done by terrestrial methods. Therefore, a combination of methods between terrestrial surveying and remote sensing techniques utilizes 
Pleiades-1B and SPOT-6 imagery to measure stand dimension variables such as crown diameter, crown closure and percentage of crown closure.

The main challenge in using high-resolution and very-high resolution imageries with visual method were the consistency and accuracy on measuring stand dimensions such as measuring the percentage of crown closure, number of trees and crown diameter [4]. To date, there is no practical algorithm available for measuring the percentage of crown cover automatically. In these challenges, the authors try to evaluate the segmentation technique to obtain information of crown cover consistently with accurate assessment. In tropical forest, particularly, each forest ecosystem type has various characteristics of canopy size, canopy layer and stand density. In this study, the segmentation analysis was evaluated on low land forest.

To analyze high spatial resolution images, the commonly used image analysis method is objectbased image analysis (OBIA), where the homogeneity of an object segmentation does not solely rely on spectral variations, but also considers the shape and size of the object [5]. The object-based method of interpreting objects considers color (spectral), size, and shape [2], [6]-[8]. Several studies have concluded that object-based methods have higher accuracy than pixel-based methods [2], [8], [9]. The weakness of the pixel-based image analysis method only considers the color (spectral) of the object that describes the brightness value (digital number) [10], [11].

The OBIA begins with a segmentation process that aims to divide the image into several homogeneous areas that do not overlap [12], [13]. The mean shift (MS) algorithm is an object-based segmentation algorithm that consists of three parameters, namely: spatial radius $(h s)$, range radius $(h r)$, and minimum region size $(M)$ [14]. Several studies have used the MS algorithm in identifying land use in the optimal GeoEye-1 composite image parameter combination (hs: 30, hr: 35, M: 85.2) [7], the segmentation studies detect forest changes due to storms on Formosat-2 red band images with optimal parameter combinations (hs: 17, hr:2) [15], the segmentation studies of pine, deciduous, and spruce trees on light detection and ranging (LiDAR) with optimal parameter combination (hs: 50, hr: 50, M: 200) [16], the segmentation study of crown closure on the Nipah ecosystem used composite images of RGB unmanned aerial vehicle (UAV) with optimal combination parameters (hs: 10, hr: 10, M: 50) [17], the segmentation studies of crown closure for the development of a stand prediction model in the SPOT-6 RGB composite image for optimal segmentation combination ( $h s: 5, h r: 21, M: 4)$ [4]. The determination of this parameter is very important because it affects the segmentation results [5], [18], [19].

Although several previous studies have been carried out in order to determine the optimal parameters for the mean shift algorithm, information on optimal parameters for wetland lowland forests is not yet available. In this study, the main objective was to find out the optimal parameters of the MS algorithm for the detection of crown closure on wetland lowland forests ecosystems using images of Pleiades-1B spatial resolution of $0.5 \mathrm{~m}$ and SPOT-6 spatial resolution of $1.5 \mathrm{~m}$.

\section{RESEARCH METHOD}

\subsection{Site description}

The research was conducted within the concession area of Tunas Timber Lestari (TTL) Inc.'s permit geographically located between 6 $38^{\prime} 40^{\prime \prime} \& 5^{\circ} 58^{\prime} 20^{\prime \prime}$ South Latitude and between $140^{\circ} 28^{\prime} 55^{\prime \prime}-141^{\circ} 9^{\prime} 15^{\prime \prime}$ East Longitude, Boven Digoel Regency, Papua Province as shown in Figure 1. Field observation and field measurements were conducted in 2018.

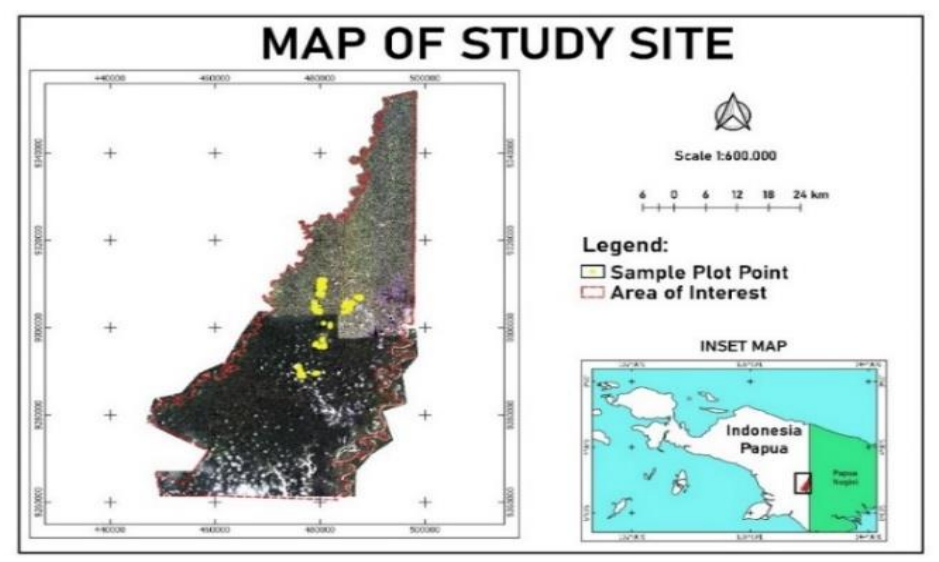

Figure 1. Map of the study site 


\subsection{Data and software}

The main data used in this study were SPOT-6 dan Pleiades-1B imagery October 11th, 2018 recording consisting of: blue, red, green, near infrared (NIR), and panchromatic bands. The tools used were global positioning system (GPS), compass, tally sheet, and camera. ERDAS Imagine 2014 version software was used for image processing, image classification and quantum geographic information system (QGIS) version 3.4.0 installed Orfeo toolbox plugins version 6.6.1 were used for image segmentation.

\subsection{Field measurement}

Field data collection used a purposive sampling method based on the percentage of canopy closure and accessibility availability. 83 sample plots in Pleiades 1B image and 49 plots were taken in the SPOT-6 image. Plot size $(50 \times 50 \mathrm{~m})$ of four sample trees measured the diameter of the crown, with the minimum diameter of a crown was of 4 meters, maximum diameter was of 10 meters, and an average diameter was of 6 meters. The size of the crown diameter became the basic knowledge to visually delineate the crown diameter above 1: 150 scale imagery.

\subsection{Pre-processing}

Preprocessing imagery consisted of (1) geometric correction (2) sharpening of Pleiades-1B and SPOT-6 multispectral images using the Brovey method [20] (3) image cropping based on area of interest (AOI) (4) RGB stacking layer Pleiades-1B images (R: green, G: red, and B: NIR) and SPOT-6 imagery (R: green, G: red, and B: blue) (5) the making of principal component analysis (PCA).

\subsection{Segmentation}

Image segmentation is the process of dividing an image into non-overlapping areas [11], [12]. The MS Algorithm is a technique of grouping images based on an iterative scheme to detect peak density (mode) using an empirical probability density function [14], [21]. This algorithm consists of three parameters (1) spatial radius $(h s)$ is the spatial radius of the object which functions to control the distance (2) radius range $(h r)$ is the spectral value range which functions as a grouping of several homogeneous pixels in one segment, (3) Minimum region size $(M)$ is the number of object pixels, the minimum size of the number of pixels to form a segment [17]. This research conducted delineation of the tree crown to determine the value $(h s)$ of the tree crown radius, $(h r)$ the spectral standard deviation of the tree crown, $(M)$ the number of pixels of the tree crown. In Tables 1 and 2 the threshold value for the combination of parameters is the minimum pixel value, maximum pixel and average pixel input parameters $(h s, h r$, and $M$ ). Thus this study tested 27 combinations of MS algorithm parameters on the blue (B), red (R), green (G), NIR bands, principal component analysis (PCA) image transformation, and Pleiades-1B composite RGB (R: green, G: red and B: NIR) and SPOT-6 RGB composite (R: green, G: red, and B: blue).

Table 1. Combination of Pleiades-1B image segmentation parameters

\begin{tabular}{|c|c|c|c|c|c|c|c|c|c|c|c|c|c|c|c|c|c|}
\hline Code & $h s$ & $\mathrm{~B}$ & $\mathrm{R}$ & $\mathrm{G}$ & $\begin{array}{l}h r \\
\text { NIR }\end{array}$ & PCA & RGB & $M$ & Code & $h s$ & B & $\mathrm{R}$ & $\mathrm{G}$ & $\begin{array}{l}h r \\
\text { NIR }\end{array}$ & PCA & RGB & $M$ \\
\hline K-01-P & 4 & 10 & 5 & 7 & 21 & 12 & 7 & 33 & K-15-P & 6 & 31 & 14 & 26 & 74 & 42 & 28 & 123 \\
\hline K-02-P & 4 & 10 & 5 & 7 & 21 & 12 & 7 & 68 & $\mathrm{~K}-16-\mathrm{P}$ & 6 & 53 & 23 & 47 & 158 & 72 & 58 & 33 \\
\hline K-04-P & 4 & 31 & 14 & 26 & 74 & 42 & 28 & 33 & K-18-P & 6 & 53 & 23 & 47 & 158 & 72 & 58 & 123 \\
\hline K-05-P & 4 & 31 & 14 & 26 & 74 & 42 & 28 & 68 & K-19-P & 10 & 10 & 5 & 7 & 21 & 12 & 7 & 33 \\
\hline K-06-P & 4 & 31 & 14 & 26 & 74 & 42 & 28 & 123 & K-20-P & 10 & 10 & 5 & 7 & 21 & 12 & 7 & 68 \\
\hline K-09-P & 4 & 53 & 23 & 47 & 158 & 72 & 58 & 123 & $\mathrm{~K}-23-\mathrm{P}$ & 10 & 31 & 14 & 26 & 74 & 42 & 28 & 68 \\
\hline K-10-P & 6 & 10 & 5 & 7 & 21 & 12 & 7 & 33 & K-24-P & 10 & 31 & 14 & 26 & 74 & 42 & 28 & 123 \\
\hline K-11-P & 6 & 10 & 5 & 7 & 21 & 12 & 7 & 68 & $\mathrm{~K}-25-\mathrm{P}$ & 10 & 53 & 23 & 47 & 158 & 72 & 58 & 33 \\
\hline K-12-P & 6 & 10 & 5 & 7 & 21 & 12 & 7 & 123 & $\mathrm{~K}-26-\mathrm{P}$ & 10 & 53 & 23 & 47 & 158 & 72 & 58 & 68 \\
\hline K-13-P & 6 & 31 & 14 & 26 & 74 & 42 & 28 & 33 & $\mathrm{~K}-27-\mathrm{P}$ & 10 & 53 & 23 & 47 & 158 & 72 & 58 & 123 \\
\hline
\end{tabular}

Note: K denotes combination; the two digit number (e.g., 22) denotes the number of combinations; P express Pleiades-1B; while B, R, G, NIR, PCA and RGB are expressing blue, red, green, near infrared, principal component, and composite of RGB; hs express spatial radius; $\mathrm{hr}$ express range radius; $\mathrm{M}$ express minimum region size 
Table 2. Combination of SPOT-6 image segmentation parameters

\begin{tabular}{cccccccccccccccccc}
\hline Code & $h s$ & B & R & G & NIR & PCA & RGB & $M$ & Code & $h s$ & B & R & G & NIR & PCA & RGB & $M$ \\
\hline K-01-S & 2 & 4 & 4 & 2 & 13 & 6 & 8 & 11 & K-15-S & 3 & 11 & 12 & 6 & 43 & 18 & 17 & 56 \\
K-02-S & 2 & 4 & 4 & 2 & 13 & 6 & 8 & 27 & K-16-S & 3 & 25 & 25 & 14 & 92 & 37 & 29 & 11 \\
K-03-S & 2 & 4 & 4 & 2 & 13 & 6 & 8 & 56 & K-17-S & 3 & 25 & 25 & 14 & 92 & 37 & 29 & 27 \\
K-04-S & 2 & 11 & 12 & 6 & 43 & 18 & 17 & 11 & K-18-S & 3 & 25 & 25 & 14 & 92 & 37 & 29 & 56 \\
K-05-S & 2 & 11 & 12 & 6 & 43 & 18 & 17 & 27 & K-19-S & 4 & 4 & 4 & 2 & 13 & 6 & 8 & 11 \\
K-06-S & 2 & 11 & 12 & 6 & 43 & 18 & 17 & 56 & K-20-S & 4 & 4 & 4 & 2 & 13 & 6 & 8 & 27 \\
K-07-S & 2 & 25 & 25 & 14 & 92 & 37 & 29 & 11 & K-21-S & 4 & 4 & 4 & 2 & 13 & 6 \\
K-08-S & 2 & 25 & 25 & 14 & 92 & 37 & 29 & 27 & K-22-S & 4 & 11 & 12 & 6 & 43 & 18 & 17 & 11 \\
K-09-S & 2 & 25 & 25 & 14 & 92 & 37 & 29 & 56 & K-23-S & 4 & 11 & 12 & 6 & 43 & 18 & 17 & 27 \\
K-10-S & 3 & 4 & 4 & 2 & 13 & 6 & 8 & 11 & K-24-S & 4 & 11 & 12 & 6 & 43 & 18 & 17 & 56 \\
K-11-S & 3 & 4 & 4 & 2 & 13 & 6 & 8 & 27 & K-25-S & 4 & 25 & 25 & 14 & 92 & 37 & 29 & 11 \\
K-12-S & 3 & 4 & 4 & 2 & 13 & 6 & 8 & 56 & K-26-S & 4 & 25 & 25 & 14 & 92 & 37 & 29 & 27 \\
K-13-S & 3 & 11 & 12 & 6 & 43 & 18 & 17 & 11 & K-27-S & 4 & 25 & 25 & 14 & 92 & 37 & 29 & 56 \\
K-14-S & 3 & 11 & 12 & 6 & 43 & 18 & 17 & 27 & & & & & & \\
\hline
\end{tabular}

Note: K denotes combination; the two digit number (e.g., 22) denotes the number of combinations; S express SPOT-6; while B, R, G, NIR, PCA and RGB are expressing blue, red, green, near infrared, principal component, and composite of RGB; hs express spatial radius; $\mathrm{hr}$ express range radius; $\mathrm{M}$ express minimum region size

\subsection{Segmentation classification}

This research combined an object-based MS and pixel-based multileaf collimator (MLC) algorithm. The MS algorithm produced object segmentation or polygons, while the MLC algorithm was used to classify images of crown closure class and gap class. Crown closure was the topmost part (branches, twigs, and leaves) of the stand, while the gap is the empty area or shadow between the branches, branches and leaves of the stand, the forest floor, and the ground surface. The results of the MLC classification were used for labeling, segmentation classification labeling process as shown in Figure 2.

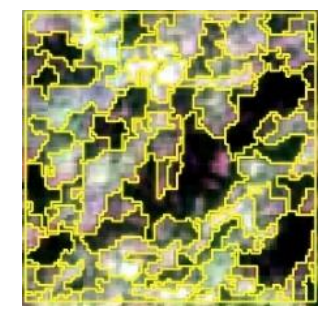

(a)

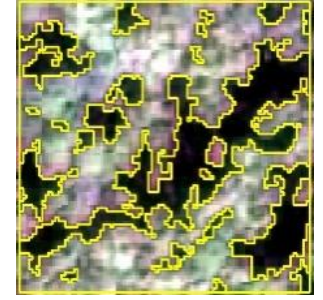

(b)

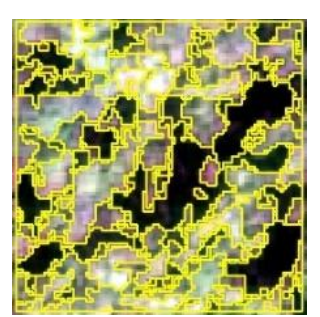

(c)

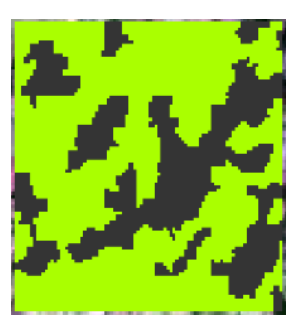

(d)

Figure 2. The steps of the segmentation classification process: (a) MS segmentation results, (b) MCL classification results, (c) The MLC and MS results overlap, and (d) segmentation classification results; $(\square)$ crown closure and $(\square)$ gap

\subsection{Accuracy assessment}

The accuracy assessment compared reference data in the form of visual delineation interpretation data on a scale of 1: 150 with segmentation data. Accuracy assessment used the area approach method [22]. The accuracy assessment procedure was reference data overlapping with segmentation data to obtain overall accuracy (OA) and kappa accuracy (KA). Accuracy assessment used the formula overall accuracy (OA) and kappa accuracy (KA) [23]:

$$
\begin{aligned}
\mathrm{OA} & =\frac{\sum_{\mathrm{i}=1}^{\mathrm{r}} \mathrm{X}_{\mathrm{ii}}}{\mathrm{N}} 100 \\
\mathrm{KA} & =\frac{\mathrm{N} \sum_{\mathrm{i}=1}^{\mathrm{r}} \mathrm{X}_{\mathrm{ii}}-\sum_{\mathrm{i}=1}^{\mathrm{r}} \mathrm{X}_{\mathrm{i}+} \mathrm{X}_{+\mathrm{i}}}{\mathrm{N}-\sum \mathrm{X}_{\mathrm{i}+} \mathrm{X}_{+\mathrm{i}}} 100
\end{aligned}
$$

where:

$$
\begin{array}{ll}
\mathrm{X}_{\mathrm{ii}} & \text { : Coincided value (number of pixel) } \\
\mathrm{X}_{+1} & : \text { The sum of column } \mathrm{j} \\
\mathrm{X}_{\mathrm{i}+} & \text { : The sum of row } \mathrm{i} \\
\mathrm{N} & \text { : Total pixel }
\end{array}
$$




\subsection{Evaluation of $\mathrm{z}$ test}

The $z$ test aims to determine whether segmentation accuracy affects band differences (red, blue, green, near infrared reflectance (NIR), principal component analysis (PCA), and RGB) and spatial images of Pleiades-1B $(0.5 \mathrm{~m})$ and SPOT-6 $(1.5 \mathrm{~m})$, the hypothesis of this study:

- Ho: The insignificant difference on the segmentation accuracy of the bands (red, blue, green, NIR, PCA, and RGB and spatial resolution of Pleiades-1B and SPOT-6 images

- H1: The significant difference on segmentation accuracy (red, blue, green, NIR, PCA, and RGB and the spatial resolution of Pleiades-1B and SPOT-6 images.

Two classification accuracy kappa values are significantly different from the criteria for a $\mathrm{z}$ score greater than $\mathrm{z}$ table of 1.96. $z$ test used the formula [23]:

$$
Z=\frac{K_{1}-K_{2}}{\sqrt{\sigma_{1}^{2}+\sigma_{2}^{2}}}
$$

where:

$$
\begin{array}{ll}
\mathrm{z} & : \text { Score } \mathrm{z} \\
K & : \text { Kappa accuracy } \\
\sigma^{2} & : \text { Kappa accuracy variance }
\end{array}
$$

\section{RESULTS AND DISCUSSION}

\subsection{The optimal combination of parameters}

The study found the best combination parameters in the Pleiades-1B and SPOT-6 images for each band as shown in Tables 3 and 4. It was found that the best parameter combination of the red band of Pleiades-1B is K-10-P-R ( $h s: 6, h r: 5, M: 33$ ) having overall accuracy (OA) of $88.93 \%$ and kappa accuracy (KA) of 73.76\%. The second-best combination was provided by the green band, i.e., K-13-P-G (hs: 6, hr: 26, $M$ : 33) with OA of $88.92 \%$ and KA of $73.55 \%$. For SPOT-6 images, the best segmentation was derived from K-01-S-RGB (hs: 2, $h r: 8, M: 11)$ giving OA of $85.72 \%$ and KA of $68.33 \%$. From the OA perspective, the OA accuracies provided by both the Pleiades and SPOT-6 images were belonged into good category [24],

\begin{tabular}{|c|c|c|}
\hline \multirow{2}{*}{ Combination $(h s$, hari \& $m$ ) } & \multicolumn{2}{|c|}{ Accuracy } \\
\hline & $\mathrm{OA}$ & KA \\
\hline K-22-P-B ( hs: 10, hari: 31, M: 33) & $88.69 \%$ & $72.91 \%$ \\
\hline K-10-P-R (hs: 6, hari: 5, M: 33) & $88.93 \%$ & $73.76 \%$ \\
\hline K-13-P-G (hs: 6, hari: 26, M: 33) & $88.92 \%$ & $73.55 \%$ \\
\hline K-10-P-NIR (hs: 6, hari: 21, M: 33) & $88.56 \%$ & $72.90 \%$ \\
\hline K-01-P-PCA (hs: 4, hari: 12, M: 33) & $87.50 \%$ & $70.31 \%$ \\
\hline K-13-P-RGB (hs: 6, hari: 28, $M: 33$ ) & $88.67 \%$ & $73.08 \%$ \\
\hline
\end{tabular}
since theirs OA values larger than $80 \%$. From the KA or Kappa value index perspective, the KA values obtained could be categorized into the moderate level, within the range of 60\%-79\%) [25].

Table 3. The optimal combination of parameters for the Pleiades-1B image

Note: $\mathrm{K}$ denotes combination; the two-digit number at the third and fourth characters (e.g., 22, $10,13,11,01 \& 13)$ denotes the number of combination; Letter "P" expresses the Pleiades-1B image; while the characters $\mathrm{B}, \mathrm{R}, \mathrm{G}, \mathrm{NIR}, \mathrm{PCA}$ and RGB are expressing the wavelength and /or band combination, namely, blue, red, green, near infrared, principal component, and composite of RGB

Table 4. The optimal combination of parameters for the SPOT-6 images

\begin{tabular}{lrr}
\hline $\begin{array}{c}\text { Combination } \\
(h s, h r \& m)\end{array}$ & OA & KA \\
\hline K-22-S-B $(h s: 4, h r: 11, M: 11)$ & $85.43 \%$ & $66.58 \%$ \\
K-13-S-R $(h s: 3, h r: 12, M: 11)$ & $85.39 \%$ & $67.22 \%$ \\
K-04-S-G $(h s: 2, h r: 6, M: 11)$ & $85.81 \%$ & $67.39 \%$ \\
K-01-S-NIR $(h s: 2, h r: 13, M: 11)$ & $84.78 \%$ & $66.15 \%$ \\
K-10-S-PCA $(h s: 3, h r: 6, M: 11)$ & $85.58 \%$ & $68.05 \%$ \\
K-01-S-RGB $(h s: 2, h r: 8, M: 11)$ & $85.72 \%$ & $68.33 \%$ \\
\hline denotes combination; the two-digit number at the third and fourth characters (e.g., 22, \\
0,13,11,01 \& 13) denotes the number of combination; Letter "S" expresses the SPOT-6 image; \\
while the characters B, R, G, NIR, PCA and RGB are expressing the wavelength and /or band \\
combination, namely, blue, red, green, near infrared, principal component and composite of RGB.
\end{tabular}




\subsection{Evaluate variations of parameter values $(h s, h r$, and $M)$}

From the analysis of both the Pleiades-1B and SPOT-6 images, it was shown that variations of spatial radius $h s$ parameter from 2 to 4 did not significantly change the accuracy, either OA or KA (Figures 3 and 4 ). The Figure 3 shows that the accuracies of natural forest crown closure segmentation derived from Pleiades images, while Figure 4, is showing the accuracies derived from SPOT-6 images. It is shown that the accuracies do not vary at all between different $h s$ values, either within the same images, or different images, for both the Pleiades-1B and SPOT-6 images. These findings are in line with the findings of some previous studies [4], [14], [17], where the variation in $h s$ parameter values did not change the classification accuracy of segmentation significantly.

Visually, the comparison between the crown closure at the original Pleiades-1B and SPOT-6 images with the segmented polygons, there is no significant difference between the segmentation results using different $h s$ parameter values. As depicted in Figure 5, the polygons of the segmentation of crown closure in the Pleiades image show that the polygons of segmentation that are the most similar in size and shape to the size dan shape of crown closure and stand gap in the original image Figure 5(a) are those generated by the $h s$ parameter with a range of values 4 and 6 Figures 5(b) and (c). Segmentation using the $h s$ parameter value of 10 tends to produce overestimated crown closure (Figure 5(d)). While the crown closure segmentation in the SPOT-6 images, the obtained polygons of the segmentation that is most similar to the size dan shape of crown closure condition in the original image Figure 5(e) is the polygon obtained using $h s$ values ranging between 2 and 3 Figures 5(f) and (g). The SPOT-6 image segmentation with a value of $h s 4$ tended to produce underestimated crown closure Figure 5(h).

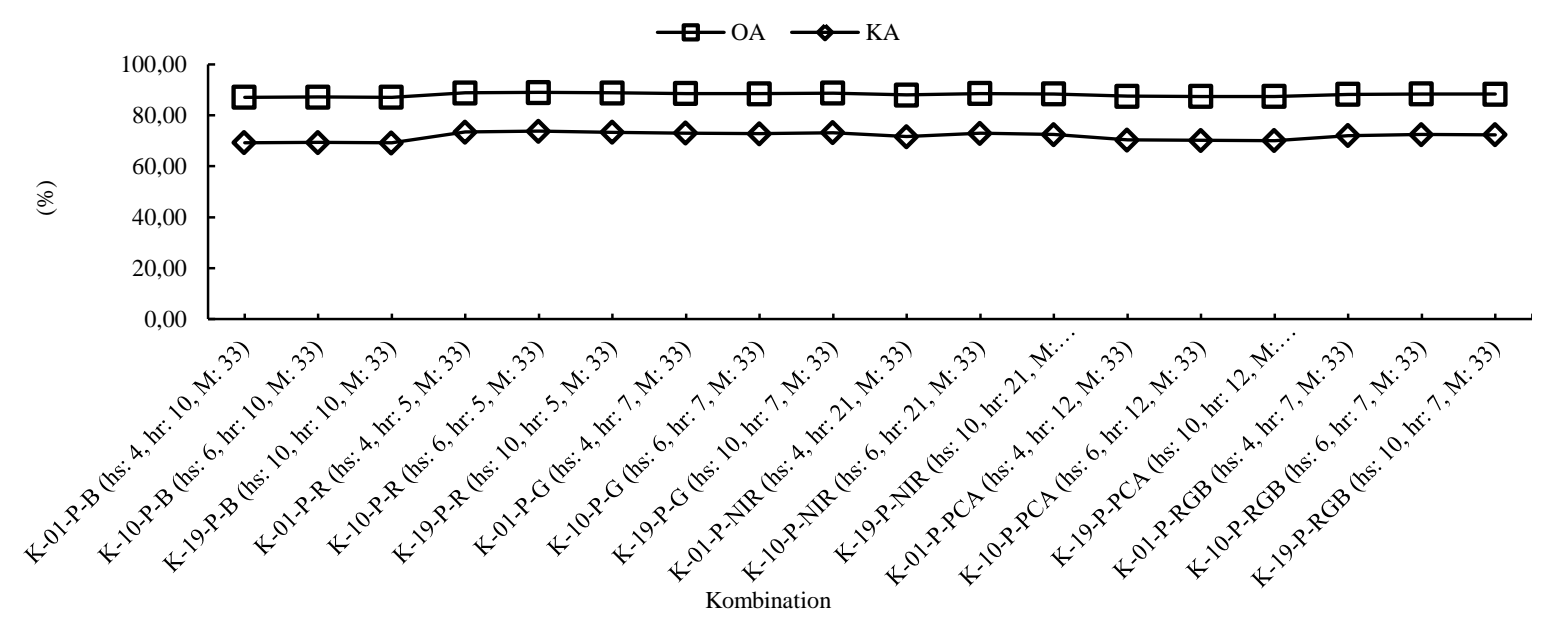

Figure 3. Variation of spatial radius $(h s)$ parameter in Pleiades-1B image

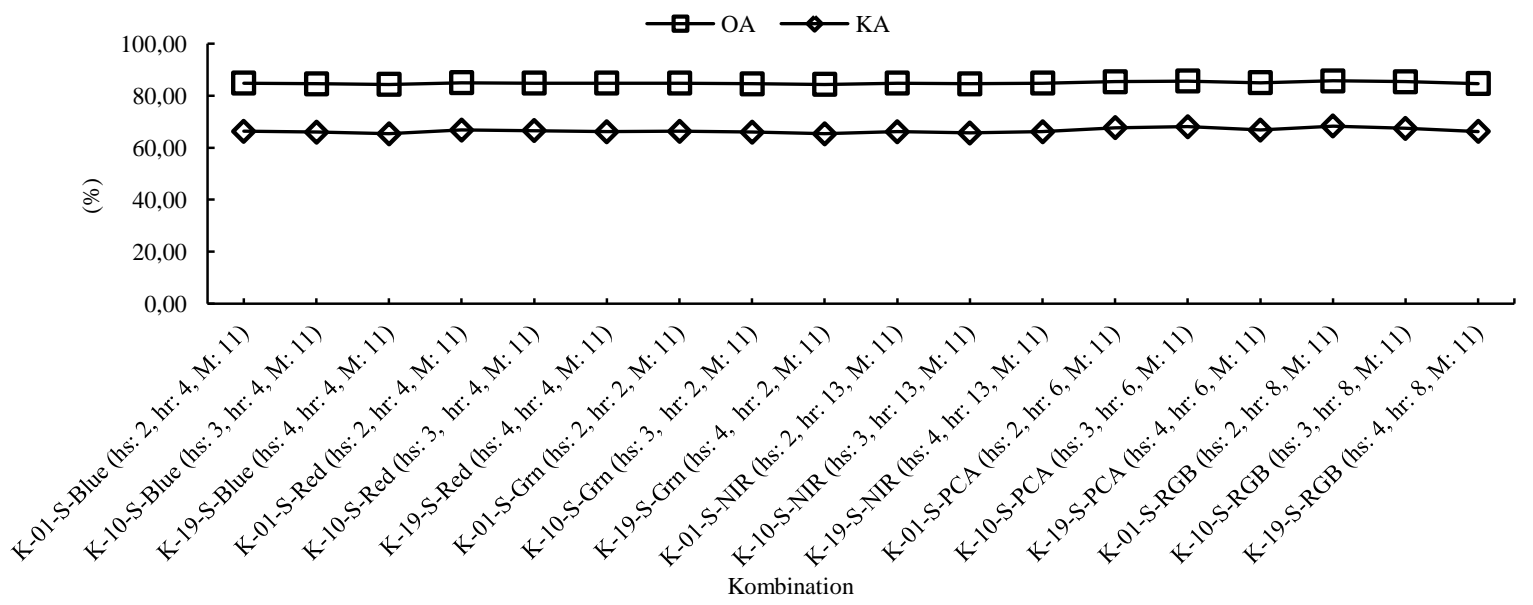

Figure 4. Variation of spatial radius $(h s)$ parameter in SPOT-6 image 


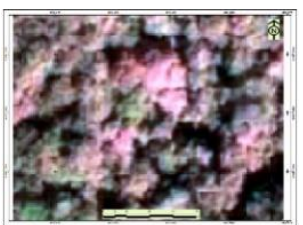

(a)

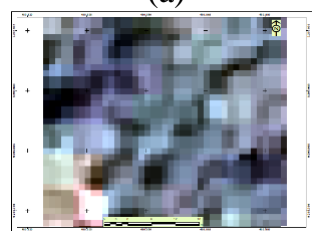

(e)

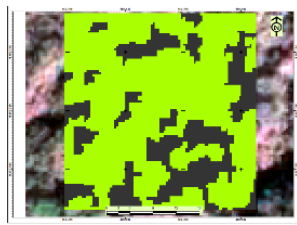

(b)

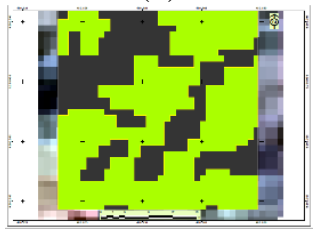

(f)

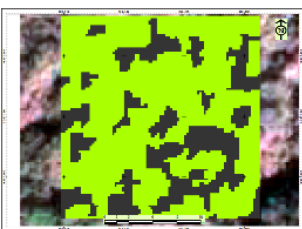

(c)

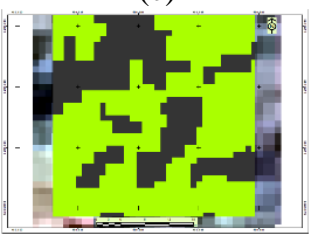

$(\mathrm{g})$

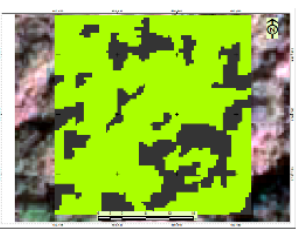

(d)

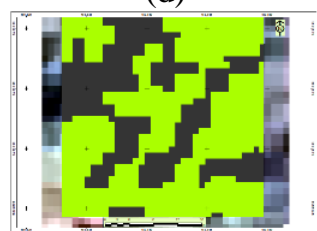

(h)

Figure 5. Display of segmentation of Pleiades-1B and SPOT-6 images using different spatial radius (hs) parameter values: (a) Original Pleiades-1B image, (b) Segmentation Pleiades-1B parameter (hs: 4), (c) Segmentation Pleiades-1B parameter $(h s: 6)$, (d) Segmentation Pleiades-1B parameter ( $h s: 10)$, (e) Original SPOT-6 image, (f) Segmentation SPOT-6 parameter ( $h s: 2)$, (g) Segmentation SPOT-6 parameter $(h s: 3)$, and (h) Segmentation SPOT-6 Segementasi $(h s: 4) ;(\square)$ crown closure and (ם) gap

Figure 5 describes how the segmentation results obtained using the Pleiades-1B and SPOT-6 images. Visually, the results of segmentation using Pleiades-1B provided more detailed canopy detection (better precision) than the results using SPOT-6 imageries. The results of stand crown closure segmentation using SPOT-6 gave a bit "rough" segmentation result compared to those provided by Pleiades, it looks like there is a "gap" generalization process. This is an indication that the detail of spatial resolution plays a significant role in gap detection, especially in detecting crown closure.

From the perspective of range radius $h r$ parameter, it was found that variations of the $h r$ parameter had been causing the high variety of both the overall and kappa accuracies Figures 6 and 7. As the $h r$ is greater, then the accuracy of OA and KA decreases, either at the Pleiades-1B or SPOT-6 images Figures 6 and 7 . The $\mathrm{OA}$ and KA accuracy values of segmentation are strongly affected by the parameter of range radius. As shown in Figures 6 and 7, the greater the $h r$, the less accurate the segmentation. In line with previous studies, the variation in the $h r$ values causes the segmentation accuracy to be low [4], [7], [15], [26], [27]. In this study, the accurate of segmentation of crown closure using Pleiades-1B were provided by $h r$ values between 7 and 42 Figure 6; while for the SPOT-6, the accurate segmentation were provided by $h r$ values ranging from 4 to 18 (Figure 7). In line with previous studies, the variation in the $h r$ values causes the segmentation accuracy to be low [4], [7], [15], [26], [27].

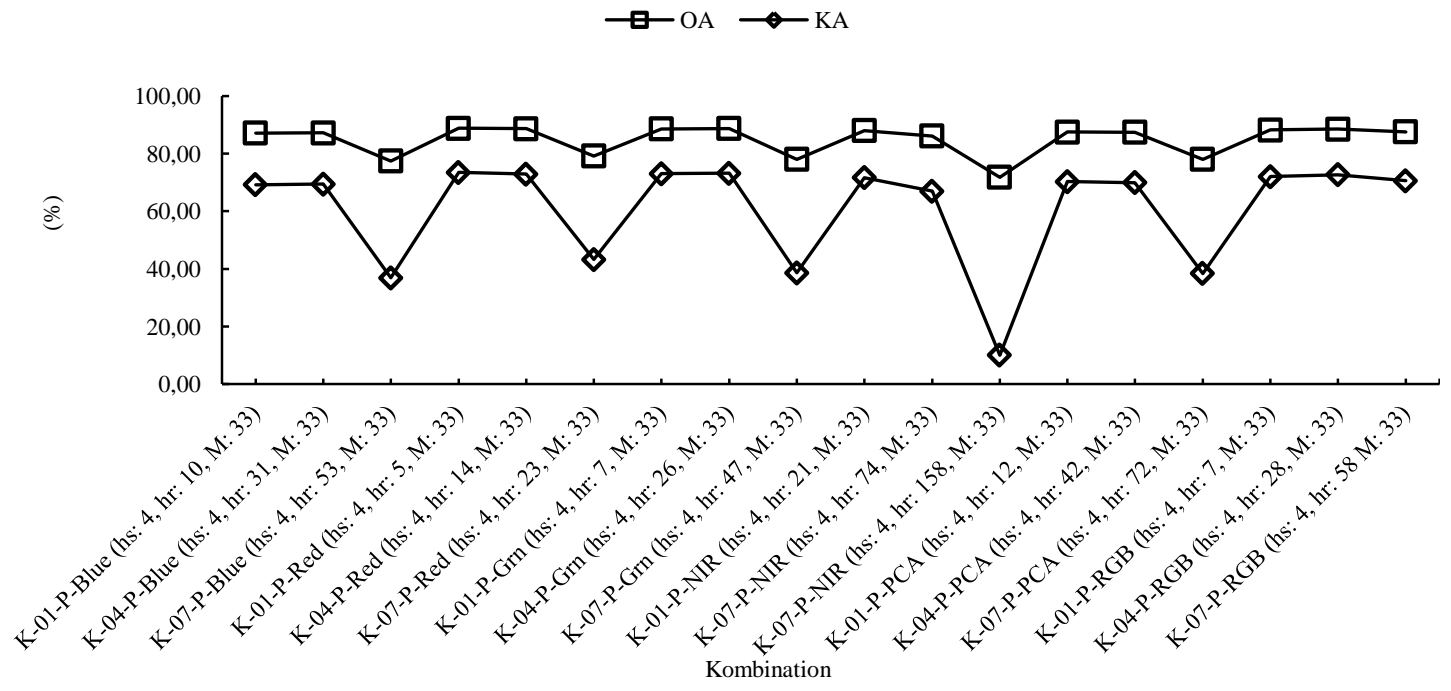

Figure 6. Variation of range radius $(h r)$ parameter in Pleiades-1B image 


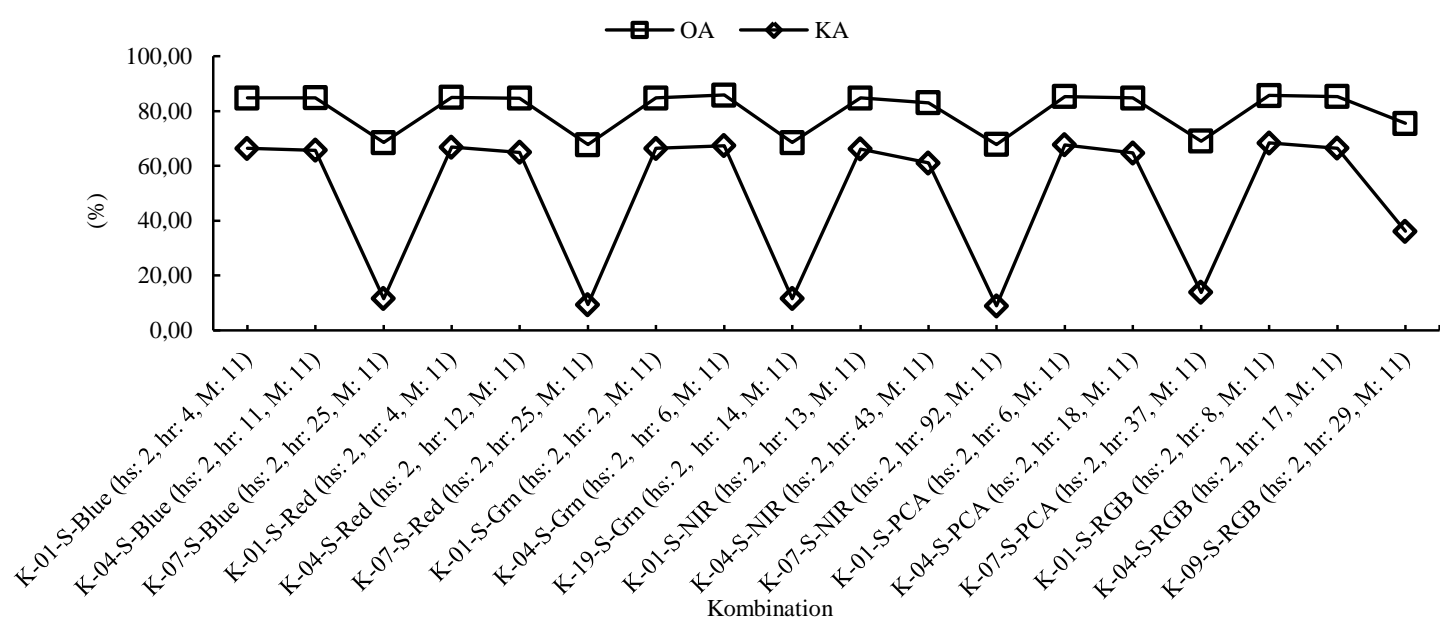

Figure 7. Variation of range radius $(h r)$ parameter in SPOT-6 image

Figure 8 shows that there is a significant spatial difference between the values of the different radius range parameters. Figure 8(a) the original Pleiades-1B image, $h r$ with a value of 21 Figure 8(b) provides the best segmentation accuracy, then the accuracy will slightly decrease when the $h r$ value is increased to 74 Figure 8(c). Furthermore, detection will be inaccurate if the $h r$ value is 158 (Figure $8(\mathrm{~d})$ ). Figure 8(e) the original SPOT-6 image, the best $h r$ parameter value is obtained when the $h r$ value of 13 Figure 8(f), better than the gap generated by the $h r$ parameter value of 43 Figure $8(\mathrm{~g})$ as well as $h r$ value of 92 Figure 8(h). In the image, the segmented SPOT-6 using an $h r$ value of 92 Figure 8(h) show that many gaps were detected as stand crown closures.

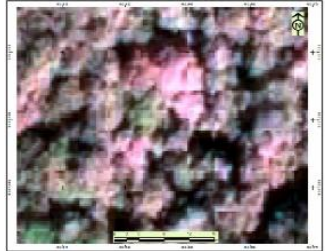

(a)

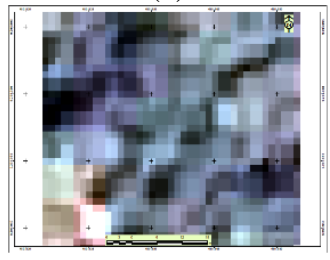

(e)

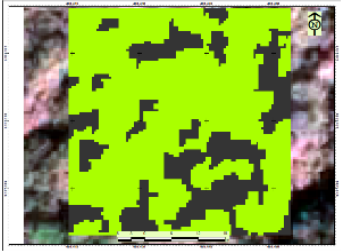

(b)

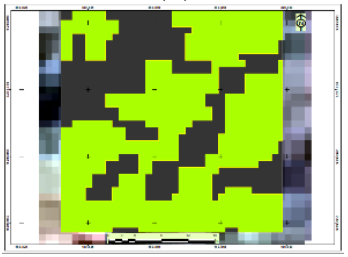

(f)

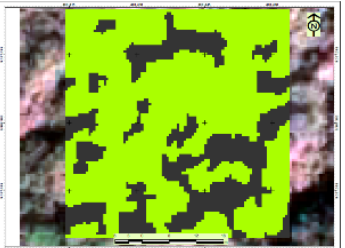

(c)

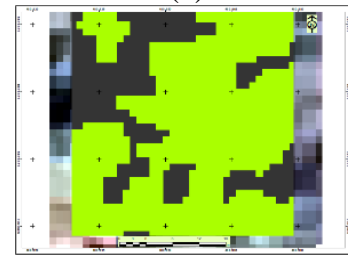

(g)

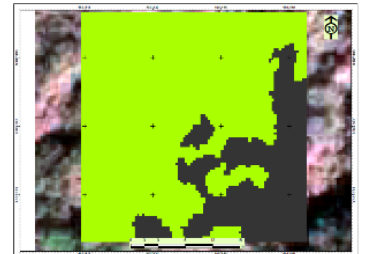

(d)

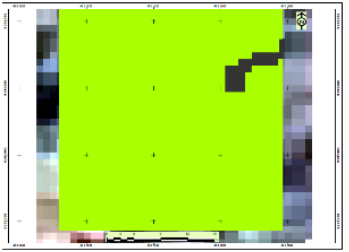

(h)

Figure 8. Display of segmentation of Pleiades-1B and SPOT-6 images using different range radius $(h r)$ parameter values: (a) Original Pleiades-1B image, (b) Segmentation Pleiades-1B parameter (hr: 21), (c) Segmentation Pleiades-1B parameter ( $h r: 74)$, (d) Segmentation Pleiades-1B parameter ( $h r: 158)$, (e) Original SPOT-6 image, (f) Segmentation SPOT-6 paramater ( $h r$ : 13), (g) Segmentation SPOT-6

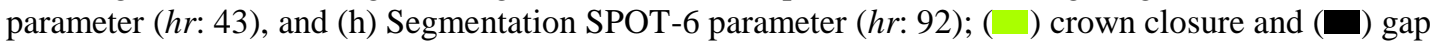

This study shows that variation of the minimum area $M$ had been caused the variation of overall accuracy (OA) and kappa accuracy (KA) (Figures 9 and 10). As shown in Figure 9, better segmentation accuracy in Pleiades-1B images is provided by $M$ value of 33, while in SPOT-6, the better accuracy was provided by $M$ value of 11 . The greater the $M$ parameter value, the less accurate the segmentation was obtained. In line with research on crown closure segmentation in the Nipah ecosystem, parameter variations $M$ lead to low segmentation accuracy [17], segmentation on land cover parameter variations $M$ has low segmentation accuracy [28]. The study results indicate that the minimum region size $M$ parameter is one of 
the key variables to successful segmentation. In this study, the $M$ parameter is very dependent on the spatial resolution of the segmented image. In the canopy closure segmentation on the Pleiades-1B image which has a spatial resolution of $0.5 \times 0.5 \mathrm{~m}$, it is found that the best $M$ parameter is around 33 pixels, equivalent to 8.25 $\mathrm{m}^{2}$. If the measure of $M$ is increased to 68 or 123 , the accuracy value will get worse (Figure 9). As for the segmentation of the canopy cover on the SPOT-6 image with a spatial resolution of $1.5 \times 1.5 \mathrm{~m}$, the best value of the $M$ parameter is 11 pixels, or equivalent to $24.75 \mathrm{~m}^{2}$, almost 3 times the size of the minimum area of the region size in Pleiades-1B. If the value of $M$ used is increased to 27 and 56, the overall accuracy value and Kappa accuracy will decrease. In the canopy cover segmentation using SPOT-6, many small canopy gaps were not detected by the SPOT-6 image, so the percentage of canopy cover became overestimated.

Spatially, the polygon variations obtained from the Pleiades-1B image segmentation are presented in Figures 11(a) to (d). From the visual comparison results, the segmentation polygon generated by parameter $M$ with a value of 11 obtained the most accurate estimation of canopy cover. If the $M$ value is further increased, the estimated canopy cover will become increasingly overestimated. For SPOT-6 images, the optimum $M$ size value is 11 , whereas if the $M$ value is increased to 27 or even 56, the accuracy value will decrease. Spatially as shown in Figures 11(a) to (h), the results of segmentation using an enlarged value will decrease the accuracy value.

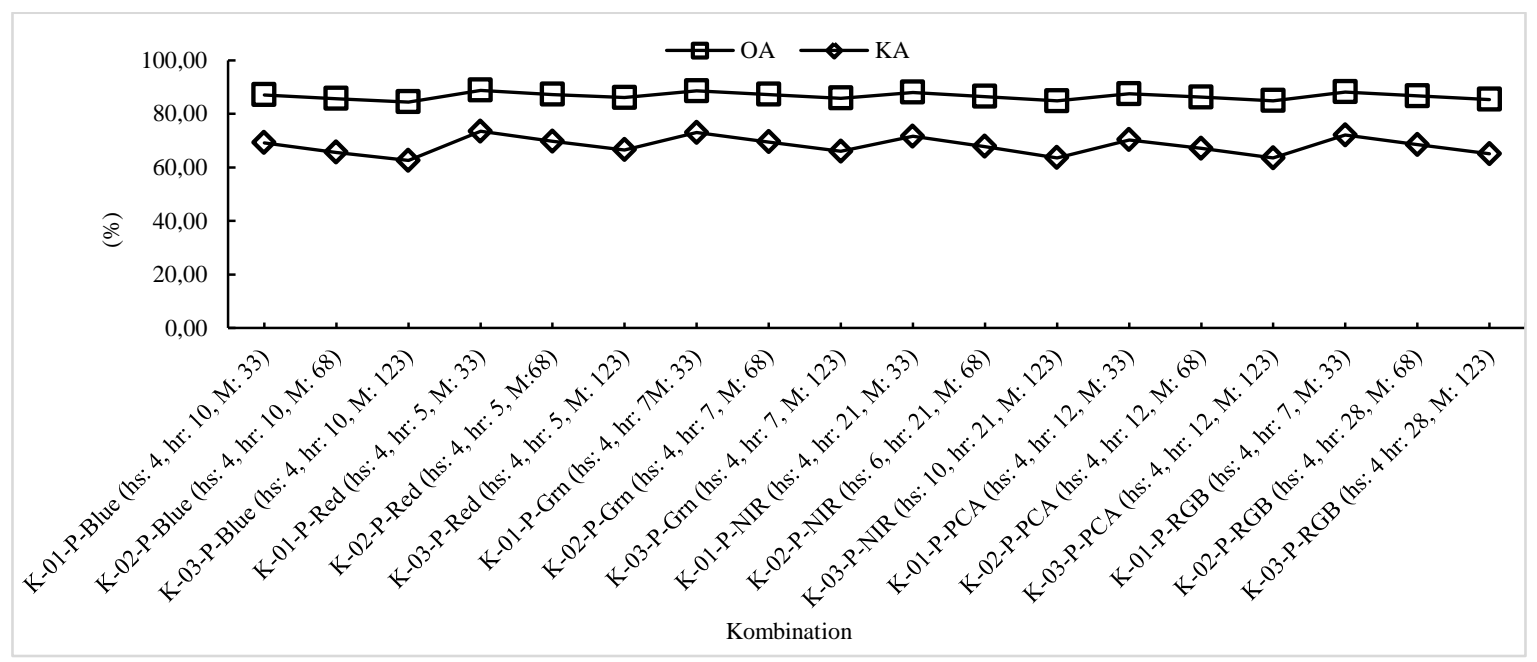

Figure 9. Variation of minimum region size $(M)$ parameter values in Pleiades-1B image

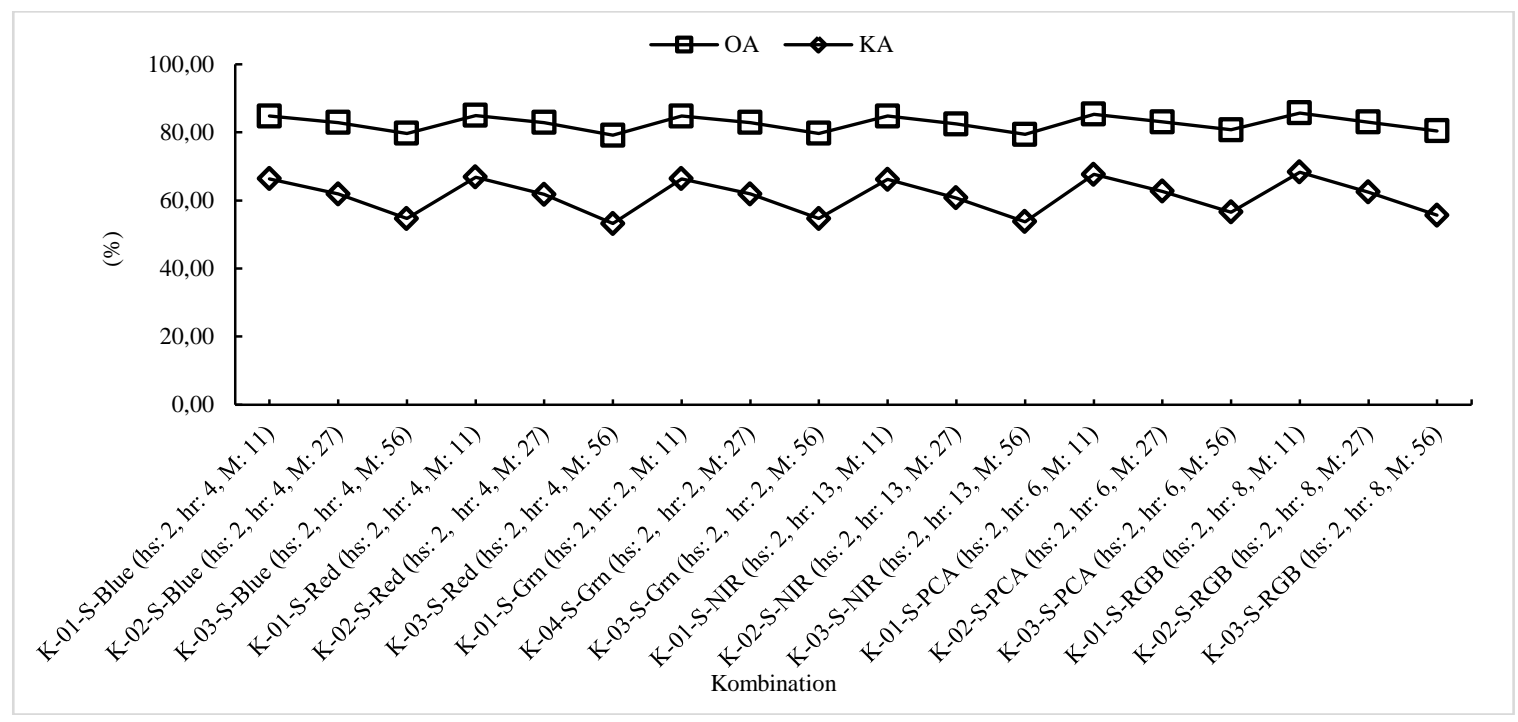

Figure 10. Variation of minimum region size $(M)$ parameter values in SPOT-6 image 


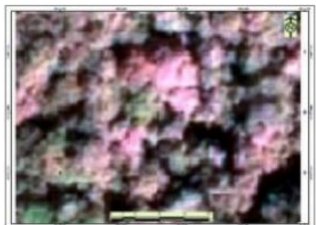

(a)

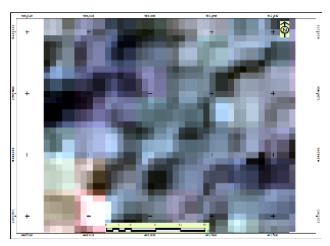

(e)

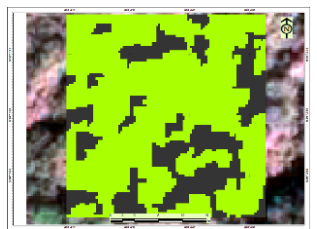

(b)

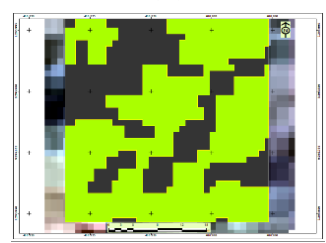

(f)

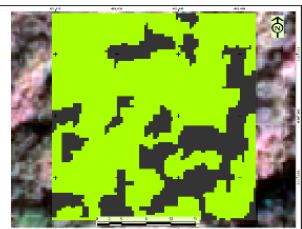

(c)

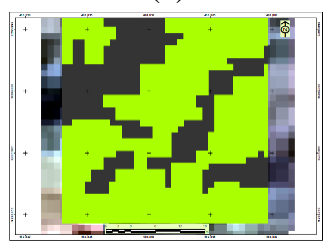

(g)

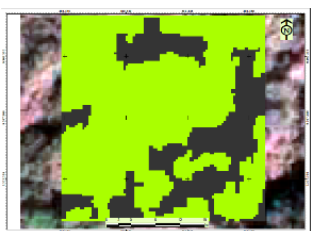

(d)

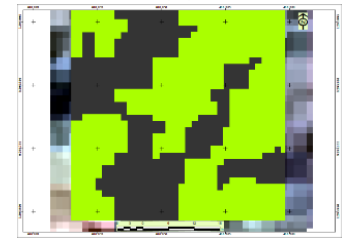

(h)

Figure 11. Display of segmentation with different parameter values $(M)$ of Pleiades-1B and SPOT-6 images: (a) Original Pleiades-1B image, (b) Segmentation Pleiades-1B parameter ( $M: 33$ ), (c) Segmentation Pleiades-

1B parameter ( $M: 68)$, (d) Segmentation Pleiades-1B parameter ( $M: 123)$, (e) Original SPOT-6 image,

(f) Segmentation SPOT-6 parameter $(M: 11)$, (g) Segmentation SPOT-6 parameter $(M: 27)$, and (h) Segmentation SPOT-6 parameter (M: 58); ( $($ ) crown closure and ( $\square)$ gap

\subsection{Comparison of segmentation accuracy between the Pleiades-1B and SPOT-6 images}

The best three of OA and KA derived from both the Pleiades-1B and SPOT-6 images, namely, K10-P-Red, K-13-P-Green, K-13-P-RGB, K-01-S-RGB, K-10-S-PCA, K-04-S-Green, we examined their significant difference by using z-test (Table 5). It is shown that there is no significant difference among the best three of segmentation derived from Pleiades. This means that the combination of K-10-P-Red, K-13-PGreen, K-13-P-RGB provide comparable results. Segmentation accuracy using the red band or the green band as well as the RGB combination is giving almost the same accuracy. This might be due to the fact than wavelength among the visible bands, where they have remarkably close inter-band correlation. Vegetation still has a relatively low reflectance in the blue and red channels compared to the green and near infrared channels [29]. The study results show that very high-resolution of Pleiades images can provide a detailed crown characteristic, such as crown closure and gap objects, even though it was segmented using only a single band such as red or green band as mentioned before.

In contrast, the study found that there are significant differences between the results obtained using very high resolution of Pleiades and high-resolution images of SPOT-6. Kappa accuracy derived from Pleiades is significantly better than those obtained from SPOT-6. The better the spatial resolution of Pleiades1B $(0.5 \times 0.5 \mathrm{~m})$ provide significantly better segmentation than those from SPOT-6 image with $1.5 \times 1.5 \mathrm{~m}$ spatial resolution (sees Table 5 at the rows and column of SPOT-6). Thus, the very high-resolution images of Pleiades-1B $(0.5 \mathrm{~m})$ produce better detection of crown closure and gap classes than the SPOT-6. The z-values with asterisk marks in Table 5 are expressing that those pairs are significantly different. In contrast, the $\mathrm{z}$ values with no asterisk mark means that those pairs have significant difference in accuracy. This study concluded that the spatial resolution affects the segmentation accuracy. Previous studies of differences in spatial resolution showed significant differences in accuracy [30]-[32].

Tabel 5. Comparison of kappa accuracy of Pleiades-1B and SPOT-6 image segmentation

\begin{tabular}{|c|c|c|c|c|c|c|c|c|c|}
\hline \multirow[b]{2}{*}{ Images } & & \multirow[b]{2}{*}{ Kappa } & \multirow[b]{2}{*}{ Variance } & \multicolumn{3}{|c|}{ Pleiades-1B } & \multicolumn{3}{|c|}{ SPOT-6 } \\
\hline & $\begin{array}{l}\text { Kappa } \\
\text { Variance }\end{array}$ & & & $\begin{array}{c}\text { K-10-P- } \\
\text { Red } \\
73.72 \\
0.12\end{array}$ & $\begin{array}{c}\text { K-13-P- } \\
\text { Green } \\
73.55 \\
0.12\end{array}$ & $\begin{array}{c}\text { K-13-P- } \\
\text { RGB } \\
73.08 \\
0.12\end{array}$ & $\begin{array}{c}\text { K-01-S- } \\
\text { RGB } \\
68.33 \\
0.23\end{array}$ & $\begin{array}{c}\text { K-10-S- } \\
\text { PCA } \\
68.05 \\
0.23\end{array}$ & $\begin{array}{c}\text { K-04-S- } \\
\text { Green } \\
67.39 \\
0.23\end{array}$ \\
\hline \multirow{4}{*}{$\begin{array}{c}\text { Pleiades- } \\
\text { 1B }\end{array}$} & & & & \multicolumn{6}{|c|}{ The z-values } \\
\hline & K-10-P-Red & 73.72 & 0.12 & \multirow{2}{*}{\multicolumn{2}{|c|}{0.44}} & 1.38 & $9.09 *$ & $9.57 *$ & $10.67 *$ \\
\hline & K-13-P-Green & 73.55 & 0.12 & & & 0.95 & $8.73 *$ & $9.20 *$ & $10.31 *$ \\
\hline & K-13-P-RGB & 73.08 & 0.12 & 1.38 & 0.95 & & $7.94 *$ & $8.42 *$ & $9.52 *$ \\
\hline \multirow{3}{*}{ SPOT-6 } & K-01-S-RGB & 68.33 & 0.23 & $9.09 *$ & $8.73 *$ & $7.94 *$ & & 0.42 & 1.38 \\
\hline & K-10-S-PCA & 68.05 & 0.23 & $9.57 *$ & $9.20 *$ & $8.42 *$ & 0.42 & & 0.96 \\
\hline & K-04-S-Green & 67.39 & 0.23 & $10.67 *$ & $10.31 *$ & $9.52 *$ & 1.38 & 0.96 & \\
\hline
\end{tabular}

Note: *) There is a significant difference in the value of $\mathrm{z}$ value $>\mathrm{z}$ table 1.96 
As presented in Table 5, it is shown that the highest Kappa value is obtained from the K-10-P-Red combination, with a KA value of $73.72 \%$. This study also shows that there is no significant difference in accuracy between K-10-P-Red and K-13-P-Green and K-13-P-RGB. The segmentation accuracy will have significantly lower accuracy if the source of segmented images has lower spatial resolution. The best parameter for detection of crown closure and gap from segmented SPOT-6 images, is K-01-S-RGB with KA $68.33 \%$. In general, it is concluded that the spectral and spatial resolution of images affect the success of the results segmentation.

The study also noted that the integration between the mean shift segmentation method and pixelbased maximum likelihood classifier (MLC) algorithms could improve the accuracy of the crown closure and gap classes detection. This is because the most critical step in segmentation is in the labelling process [17], [33]. The MLC algorithm is a pixel-based classification based on spectral responses taken from the training area [11]. However, since high-spatial resolution imagery could has higher spectral variation in a object, then it will reduce the accuracy, when the classification is solely based upon the pixel-based algorithms alone [8]. Thus, the interactive hybrid between the object-based and pixel-based approach is recommended.

\section{CONCLUSION}

From the foregoing discussion, the study concluded that the most accurate combination of segmentation parameters for detecting crown closure using Pleiades-1B image in wetland forest ecosystems is a combination of parameters of ( $h s: 6, h r: 5, M: 33)$ with an OA of $88.9 \%$ and KA of $73.8 \%$. For segmenting crown closure using SPOT-6, the best combination of parameters is (hs: 2, hr: $8, M: 11$ with an accuracy of $85.6 \% \mathrm{OA}$ and $68.3 \%$. It is also concluded that there is no significant difference between the segmentation using single band, composite, or synthetic principal component analysis (PCA) for each image using either Pleiades-1B or SPOT-6 images. Pleiades-1B with has a very high spatial resolution provide higher segmentation accuracy, better than the SPOT-6 (with $1.5 \mathrm{~m}$ spatial resolution). In general, the application of MS algorithm for detecting crown closure in Pleiades provides good overall accuracy of $85 \%$. The study still leave a future work for evaluating various spatial and spectral resolutions including the images derived from UAV with sub-meter spatial resolution. Verification of models in diverse types of ecosystems also needs to be continued to examine its stability and measure whether this model could be generalized.

\section{ACKNOWLEDGEMENTS}

The authors would like to extend high appreciation to the Board of Directors of Tunas Timber Lestari (TTL) Inc., Korindo Group, for allowing the authors to use the Pleiades-1B and SPOT-6 imageries of the concession area for supporting the implementation of the research. Sincerely thanks are also extended to the Head of the Laboratory of Forest Resource Inventory and Remote Sensing, IPB University for data analysis support and all colleagues for ground data collection in Papua.

\section{REFERENCES}

[1] I. N. S. Jaya et al., "The Examination of The Satellite Image-Based Growth Curve Model Within Mangrove Forest," Jurnal Manajemen Hutan Tropika, vol. 25, no. 1, pp. 44-50, 2019, doi: 10.7226/jtfm. 5.1.44.

[2] T. Blaschke, "Object Based Image Analysis for Remote Sensing," ISPRS Journal of Photogrammetry and Remote Sensing, vol. 65, no. 1, pp. 2-16, Jan. 2010, doi: 10.1016/j.isprsjprs.2009.06.004.

[3] A. Kuliešis, et al., "Using Continuous Forest Inventory Data for Control of Wood Production and Use in Large Areas: A Case Study in Lithuania," Forests, vol. 11, no. 10, 2020, doi:10.3390/f11101039.

[4] D. P. Apriyanto, I. N. S. Jaya, and N. Puspaningsih, "Examining the Object-Based and Pixel-Based Image Analyses for Developing Stand Volume Estimator Model," Indonesian Journal of Electrical Engineering and Computer Science (IJEECS), vol. 15, no. 3, pp. 1586-1596, Sep. 2019, doi: 10.11591/ijeecs.v15.i3.pp1586-1596.

[5] M. D. Hossain and D. Chen, "Segmentation for Object-Based Image Analysis (OBIA): A review of algorithms and challenges from remote sensing perspective," ISPRS Journal of Photogrammetry and Remote Sensing, vol. 150, pp. 115-134, Apr. 2019, doi: 10.1016/j.isprsjprs.2019.02.009.

[6] T. Lillesand, R. W. Kiefer, and J. Chipman, "Concepts and Foundation of Remote Sensing," in Remote Sensing and Image Interpretation, Flahive R, Eds. 7, New York, NY: Wiley, 2015.

[7] Y. G. Byun, Y. K. Han, and T. B. Chae, "A multispectral Image Segmentation Approach for Object-Based Image Classification of High Resolution Satellite Imagery," KSCE Journal of Civil Engineering, vol. 17, no. 2, pp. 486-497, Mar, 2013, doi: 10.1007/s12205-013-1800-0.

[8] T. Blaschke et al., "Geographic Object-Based Image Analysis-Towards a new Paradigm," ISPRS Journal of Photogrammetry and Remote Sensing, vol. 87, pp. 180-191, Jan. 2014, doi: 10.1016/j.isprsjprs.2013.09.014.

[9] E. A. Addink, F. M. B. Van Coillie, and S. M. De Jong, "Introduction to the GEOBIA 2010 special issue: From pixels to geographic objects in remote sensing image analysis," International Journal of Applied Earth Observation and Geoinformation, vol. 15, pp. 1-6, Apr, 2012, doi: 10.1016/j.jag.2011.12.001. 
[10] L. D. Robertson and D. J. King, "Comparison of Pixel-and Object-Based Classification in Land Cover Change Mapping," International Journal of Remote Sensing, vol. 32, no. 6, pp. 1505-1529, Mar. 2011, doi: 10.1080/01431160903571791.

[11] S. W. Myint, P. Gober, A. Brazel, S. Grossman-Clarke, and Q. Weng, "Per-pixel vs. Object-Based Classification of Urban Land Cover Extraction Using High Spatial Resolution Imagery," Remote Sensing of Environment, vol. 115, no. 5, pp. 1145-1161, May. 2011, doi: 10.1016/j.rse.2010.12.017.

[12] R. Bergman and H. Nachlieli, "Perceptual Segmentation: combining image aegmentation with object tagging," IEEE Transactions On Image Processing, vol. 20, no. 6, pp. 1668-1680, Jun. 2011, doi: 10.1109/TIP.2010.2088970.

[13] M. W. Khan, "A Survey: Image Segmentation Techniques," International Journal of Future Computer and Communication, vol. 3, no. 2, pp. 89-93, Apr, 2014, doi: 10.7763/ijfcc.2014.V3.274.

[14] D. Comaniciu and P. Meer, "Mean Shift: a robust approach toward feature space analysis," IEEE Transactions on Pattern Analysis and Machine Intelligence, vol. 24, no. 5, pp. 603-619, May 2002, doi: 10.1109/34.1000236.

[15] N. Chehata, C. Orny, S. Boukir, D. Guyon, and J. P. Wigneron, "Object-Based Change Detection in Wind StormDamaged Forest Using High-Resolution Multispectral Images," International Journal of Remote Sensing, vol. 35, no. 13, pp. 4758-4777, Jul. 2014, doi: 10.1080/01431161.2014.930199.

[16] Z. Wu, V. Heikkinen, M. Hauta-Kasari, J. Parkkinen and T. Tokola, "ALS Data Based Forest Stand Delineation with a Coarse-to-Fine Segmentation Aproach," 2014 7th International Congress on Image and Signal Processing, 2014, pp. 547-552, doi: 10.1109/CISP.2014.7003840.

[17] R. P. Silalahi, I. N. S. Jaya, T. Tiryana, and F. Mulia, "Assessing the Crown Closure of Nypa on UAV Images Using Mean-Shift Segmentation Algorithm," Indonesian Journal of Electrical Engineering and Computer Science (IJEECS), vol. 9, no. 3, pp. 722-730, Mar. 2018, doi: 10.11591/ijeecs.v9.i3.pp722-730.

[18] J. Stefanski, B. Mack, and O. Waske, "Optimization of Object-Based Image Analysis With Random Forests for Land Cover Mapping," in IEEE Journal of Selected Topics in Applied Earth Observations and Remote Sensing, vol. 6, no. 6, pp. 2492-2504, Dec. 2013, doi: 10.1109/JSTARS.2013.2253089.

[19] I. Dronova, "Object-Based Image Analysis in Wetland Research: A Review," Remote Sensing, vol. 7, no. 5, pp. 6380-6413, May 2015, doi: 10.3390/rs70506380.

[20] B. Johnson, "Effects of Pansharpening on Vegetation Indices," ISPRS Int. J. Geo-Inf. vol. 3, no. 2, pp. 507-522, 2014, doi:10.3390/ijgi3020507.

[21] J. N. Kaftan, A. A. Bell, and T. Aach, "Mean Shift Segmentation Evaluation of Optimization Techniques," in ' 08 Proceedings of the Third International Conference on Computer Vision Theory and Applications, 2008, pp. 365-374, doi: 10.5220/0001085003650374.

[22] M. Neubert, H. Herold, and G. Meinel, "Assessing Image Segmentation Quality-Concepts Methods and Application," in Object-Based Image Analysis, 2008, pp. 769-784, doi: 10.1007/978-3-540-77058-9_42.

[23] G. Rosenfield and K. Fitzpatrick-Lins, "A Coefficient of Agreement as a Measure of Thematic Classification Accuracy," Photogrammetric Engineering and Remote Sensing, vol. 52, no. 2, pp. 223-227, 1986.

[24] J. R. Thomlinson, P. V. Bolstad, and W. B. Cohen, "Coordinating Methodologies for Scaling Landcover Classifications from Site-Specific to Global: Steps toward validating global map products," Remote Sensing of Environment, vol. 70, no. 1, pp. 16-28, Oct. 1999, doi: 10.1016/S0034-4257(99)00055-3.

[25] M. L. McHugh, "Interrater Reliability: the kappa statistic," Biochemia Medica, vol. 22, no. 3, pp. 276-282, 2012, doi: 10.11613/BM.2012.031.

[26] D. Ming, J. Li, J. Wang, and M. Zhang, "Scale Parameter Selection by Spatial Statistics for GeOBIA: Using meanshift based multi-scale segmentation as an example," ISPRS Journal of Photogrammetry and Remote Sensing, vol. 106, pp. 28-41, Aug. 2015, doi: 10.1016/j.isprsjprs.2015.04.010.

[27] D. Demirović, "An Implementation of the Mean Shift Algorithm," Image Processing On Line, vol. 9, pp. 251-268, Sep. 2019, doi: 10.5201/ipol.2019.255.

[28] Y. Qiu, D. Ming, and X. Zhang, "Object Oriented Land Cover Classification Combining Scale Parameter Preestimation and Mean-Shift Segmentation," 2016 IEEE International Geoscience and Remote Sensing Symposium (IGARSS), 2016, pp. 6332-6335, doi: 10.1109/IGARSS.2016.7730655.

[29] Y. Xie, Z. Sha, and M. Yu, "Remote sensing imagery in vegetation mapping: a review," Journal of Plant Ecology, vol. 1, no. 1, pp. 9-23, 2008, doi: 10.1093/jpe/rtm005.

[30] N. Mesner and K. Oštir, "Investigating the Impact of Spatial and Spectral Resolution of Satellite Images on Segmentation Quality," Journal of Applied Remote Sensing, vol. 8, no. 1, pp. 1-14, Jan. 2014, doi: 10.1117/1.Jrs.8.083696.

[31] A. Š. Lenarčič, K. Ritlop, N. Đurić, K. Čotar, and K. Oštir, "Impact of Spatial Resolution on Correlation Between Segmentation Evaluation Metrics and Forest Classification Accuracy," in Image and Signal Processing for Remote Sensing XXI, Oct. 2015, pp. 1-16, doi:10.1117/12.2194794.

[32] T. Kavzoglu and H. Tonbul, "Segmentation Quality Assessment for Varying Spatial Resolutions of Very High Resolution Satellite Imagery," in '18 Eurasian GIS Congress, 2018.

[33] A. Morales-Gonz'alez, E. Garc'1a-Reyes and L. E. Sucar, "Unsupervised Segmentation Evaluation for Image Annotation," in '15 Proceedings of the 10th International Conference on Computer Vision Theory and Applications, Mar. 2015, pp. 148-155, doi: 10.5220/0005314201480155. 


\section{BIOGRAPHIES OF AUTHORS}
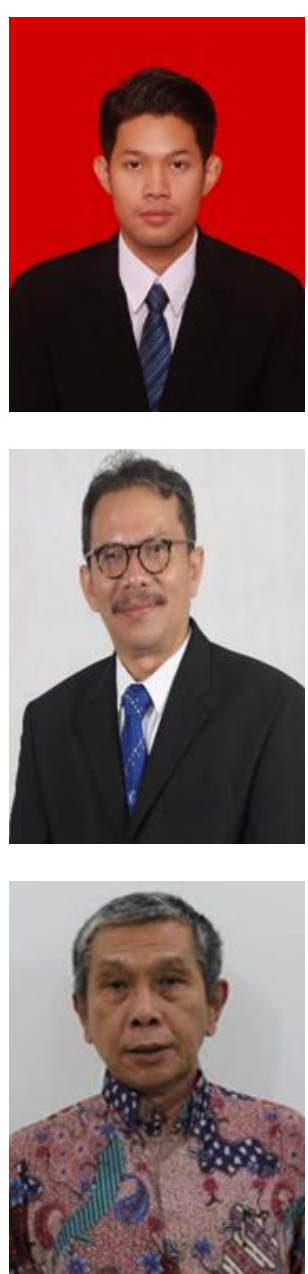

Beni Iskandar is a postgraduate student of Forest Management Science, Graduate School of IPB University. His research interest is in remote sensing and image segmentation to support forest planning activity. He got his bachelor degree in forestry in 2017 from Faculty of Forestry, Lambung Mangkurat University.

Prof. Dr. I Nengah Surati Jaya is a permanent professor since 2007 at the Faculty of Forestry and Environment, IPB University, majoring in applied remote sensing and spatial geoscience. Since 1990, he had numerous researches, publications and works related to the remote sensing and spatial modelling in the forestry and environmental related sectors. Recently, his research has been focused on the use high resolution images, terrestrial data, and fish-eye-camera based data to develop practical method as well as algorithm to support a sustained forest management. He got his PhD in 1996 from Niigata University, Master's degree in 1993 from Graduate School of Environmental Science, Niigata University, Bachelor of Forestry in 1985 from Forest Management Department, Faculty of Forestry, IPB University.

Dr. Muhammad Buce Saleh is a permanent associate professor at the Forest Management Department, Faculty of Forestry and Environment, IPB University. His special interest is in the field of remote sensing and geomatics in forest management. He got his $\mathrm{PhD}$ in 2002 from Niigata University, Master of Sciences in 1988 from Graduate School of Bandung Institute of Technology and Bachelor of Forestry in 1981 from Forest Management Department, Faculty of Forestry IPB university. 